Musées, Patrimoine et Culture scientifiques et techniques

$157 \mid 2015$

janvier-février 2015

\title{
Visiter un musée de beaux-arts en famille : difficultés et stratégies parentales
}

Aude Joly

\section{OpenEdition}

\section{Journals}

Édition électronique

URL : https://journals.openedition.org/ocim/1480

DOI : $10.4000 /$ ocim. 1480

ISSN : 2108-646X

Éditeur

OCIM

Édition imprimée

Date de publication : 1 janvier 2015

Pagination : 25-31

ISSN : 0994-1908

Référence électronique

Aude Joly, « Visiter un musée de beaux-arts en famille : difficultés et stratégies parentales », La Lettre de I'OCIM [En ligne], 157 | 2015, mis en ligne le 01 janvier 2016, consulté le 21 septembre 2021. URL : http://journals.openedition.org/ocim/1480 ; DOI : https://doi.org/10.4000/ocim.1480

Ce document a été généré automatiquement le 21 septembre 2021.

Tous droits réservés 


\section{Visiter un musée de beaux-arts en famille : difficultés et stratégies parentales}

Aude Joly

Visiteurs en famille au musée Fabre, Montpellier Agglomération.

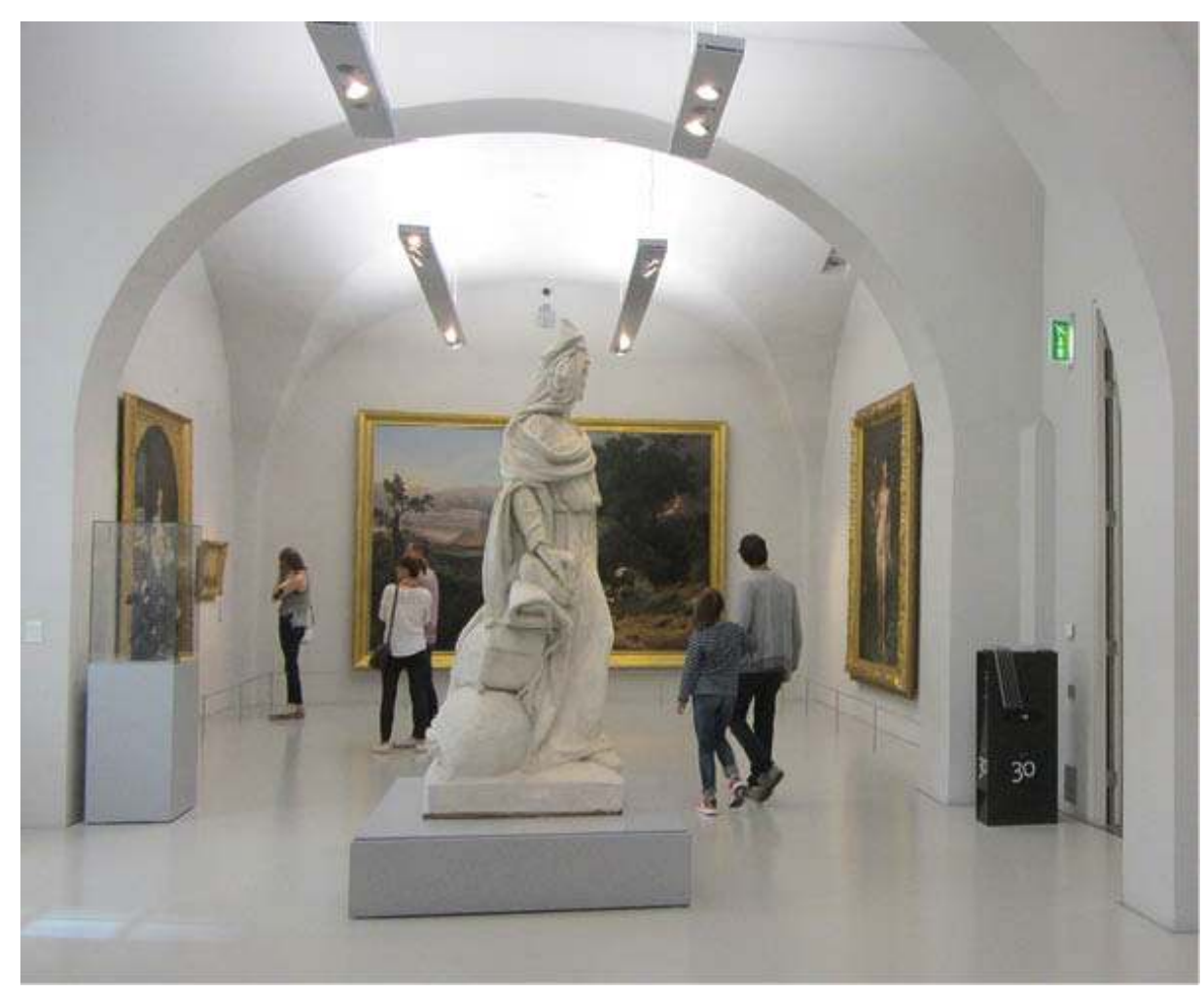

(C) Aude Joly 
1 S'il est moins présent que dans les autres types de musées, le public famille est loin d'être négligé par les musées de beaux-arts français qui développent à son intention une offre de médiation variée (visites, ateliers, livrets, audio-guides, dispositifs numériques...). Toutefois, peu d'études à ce jour font état de l'impact que ce mode d'accompagnement particulier peut avoir sur l'expérience de visite et la réception des œuvres exposées (Pasquier, 2005). Pourtant, visiter un musée de beaux-arts en famille, c'est avant tout le visiter accompagné: de ses enfants lorsqu'on est parent; de ses parents quand on est enfant. On peut dès lors se demander en quoi la visite en famille diffère d'une visite solitaire ou entre amis. Que se passe-t-il lorsque parent et enfant ${ }^{1}$ observent ensemble une œuvre d'art? Quels types de comportements adoptent-ils l'un envers l'autre?

\section{Le statut du "parent médiateur"}

2 Lors d'une visite en famille, De Singly reconnaît à l'adulte avant tout le rôle d'accompagnateur, considérant qu'il "est dans la situation avec surtout son identité statutaire [...] en tant que parent, alors que l'autre [l'enfant] vit cela avec son identité personnelle" (de Singly, 2003). Le rapport de l'adulte à l'enfant se rapprocherait ainsi d'une relation correspondant à un rapport maître/élève, relation qui peut être vécue, comme une pression éducative par l'adulte, qui se sent "investi d'une mission de transmission pédagogique " (Debenedetti, 2003). Qu'il soit conscient ou non, ce processus de transmission serait indissociable de la pratique de visite en famille, moment particulièrement propice à des échanges entre générations centrés sur la transmission d'éléments provenant d'un double héritage: celui du milieu familial et celui représenté par les collections du musée (Cordier, 1997). La transmission intergénérationnelle, notamment dans le domaine de la culture, est en outre largement considérée comme un processus vertical et descendant (c'est-à-dire des parents vers l'enfant) (Octobre, 2008). Ce désir de transmission (d'un goût, d'une pratique, d'une culture) du parent vers l'enfant semble d'autant plus présent lors des visites en famille de musées de beaux-arts, institution pour lesquelles les visites en famille se font majoritairement à l'initiative des parents et dans une logique davantage éducative que dans les musées présentant d'autres types de collections (Jonchery, 2005).

Par ailleurs, l'étude de Jonchery qui compare les logiques de visite du public famille de trois musées parisiens (dont un musée de beaux-arts) montre que commencer sa "carrière" de visiteur en famille par des musées présentant des collections d'art serait plus difficile que de construire son expérience en commençant par la visite d'autres types de collections. En effet, explique-t-elle, la visite en famille engendre l'apprentissage d'un mode de visite différent du mode individuel. "La tendance première et naturelle consiste [...] à copier le modèle du visiteur adulte ce qui entrainent une lassitude rapide des enfants notamment dans les musées d'art" (Jonchery, 2005). La visite en famille d'un musée de beaux-arts semblerait alors une activité peu aisée de prime abord.

4 En partant de ce double constat de la prédominance d'une logique de transmission éducative et de la relative difficulté pour les familles à accéder à une expérience de visite satisfaisante pour petits et grands, cet article a pour objectif de rendre compte de certaines difficultés auxquelles les parents accompagnés de leur(s) enfant(s) peuvent être confrontés dans des salles d'exposition. La principale hypothèse étant que la posture d'accompagnateur de l'adulte, que nous qualifierons de "parent-médiateur", 
provoquerait, lorsque celui-ci interagit dans un musée de beaux-arts avec son enfant, des situations rendant la situation de transmission difficiles à assumer.

Pour faire avancer les connaissances en cette matière, une étude de terrain a été menée en décembre 2012 dans l'exposition permanente du musée Fabre de Montpellier. À notre invitation, 26 familles composées d'enfants âgés de 7 à 11 ans et de leur(s) parent(s), sont venues visiter le musée ${ }^{2}$ Il s'agit de familles présentant des profils culturels et socioéconomiques très différents: si certaines fréquentent assidûment les musées, d'autres pénétraient pour la première fois dans un musée de beaux-arts. Parents et enfants étaient invités à visiter de manière autonome et à leur rythme le deuxième étage du musée, présentant entre autres des œuvres de Pierre Soulages, Nicolas de Staël, Germaine Richier, Frédéric Bazille et Gustave Courbet. Par ailleurs, les familles ne disposaient d'aucune autre forme de médiation que la mise en exposition elle-même, incluant les textes présents dans l'espace, à savoir les cartels et des fiches de salles. Volontairement, nous ne leur avons pas remis les livrets et les audio-guides généralement proposés aux familles.

Un père et son fils dans les salles consacrées au peintre Soulages au musée Fabre, Montpellier Agglomération.

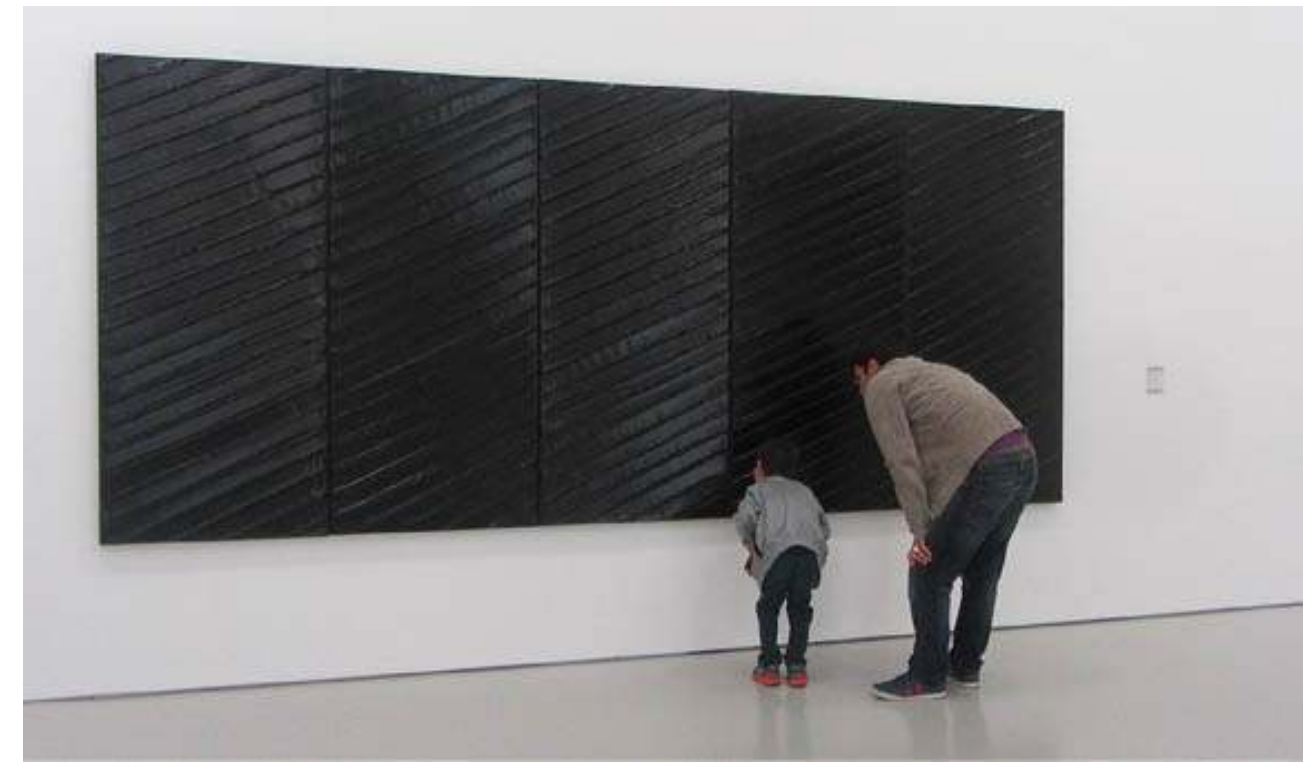

(C) Aude Joly

La visite était suivie d'un entretien semi-directif mené par la chercheure et portant sur leurs habitudes de visite en famille et sur l'expérience qu'ils venaient de vivre ensemble. Les questions étaient orientées de telle sorte que les difficultés rencontrées par les parents lors de cette expérience (mais aussi à l'occasion de visites antérieures) soient évoquées.

Les difficultés exprimées résultent essentiellement de la position de parent-médiateur assumée par l'adulte lors d'une visite en famille. En fonction de sa familiarité avec la pratique muséale, de ses intentions de visites, de ses connaissances, le parent les perçoit de manière plus ou moins pressante et développe des stratégies de contournement. Il est toutefois important de noter que si nombre de difficultés ont été exprimées par les parents, tous ont apprécié cette expérience partagée avec leur enfant et en font un retour positif. 


\section{Respecter les codes de comportements}

8 Visiter un musée de beaux-arts, c'est tout d'abord, pour le parent accompagné de son enfant, se confronter à un espace régi par des règles et au personnel de surveillance chargé les faire respecter. Pour certains parents rencontrés, le musée de beaux-arts est perçu comme un lieu peu adapté à l'accueil des jeunes enfants. Le silence qui y règne, les codes de comportement (ne pas toucher, ne pas courir, ne pas crier) qui y sont associés conduisent certains des parents à appréhender le comportement de leur enfants (Adams et Moussouri, 2002). À la question "Visitez-vous différemment un musée lorsque vous le faites avec votre enfant", les réponses spontanées de ces deux mères, qui fréquentent pourtant régulièrement les musées avec leurs enfants de 7 et 8 ans, sont révélatrices de la mission de transmission d'une attitude de visite qu'elles adoptent : "Ah oui! Déjà, je surveille qu'il ne touche pas" (mère de L. 8 ans). "C'est d'abord surveiller qu'ils ne touchent pas, c'est vrai qu'il faut être sur ses gardes" (mère de R. 7 ans). Prévoir une visite de musée de beaux-arts peut ainsi générer chez l'adulte une certaine inquiétude. Les parents sont responsables du bon comportement de l'enfant, et semblent particulièrement sensibles à la fois à la présence des gardiens et au regard des autres visiteurs. Le champ lexical, parfois proche de celui de la peur, employé par les parents interrogés, souligne leur sentiment de n'être pas tout à fait à leur place. La visite se teinte d'un caractère stressant, on craint "qu'un gardien ne surgisse" :

$\mathrm{M}^{3}$ : C'est vrai qu'on se sent observés, c'est normal...

I : Vous pensez que cela est dû à la présence des enfants?

M : Ah oui, parce que j'étais avec les enfants, c'est sûr [...] c'est vrai qu'on ne se sent pas détendu"

(mère de A. 9 ans et R. 7 ans)

Cette crainte semble trouver sa source davantage dans des expériences de visites passées difficiles (où il a par exemple été demandé aux parents de sortir car leur jeune enfant était trop bruyant), et toucher essentiellement des familles qui visitent peu les musées, mais dans une démarche essentiellement éducative. Si la pression liée au bon comportement de l'enfant est présente, elle participe aussi à l'objectif pédagogique de la visite : "on a eu justement l'occasion d'en parler. Je lui ai dit: si tu veux chanter, danser, rigoler parler fort et boire un coup, tu vas en discothèque! Si tu veux rentrer dans un musée, [...] tu te tais, c'est comme à la bibliothèque, là tu viens voir de l'art" (mère de M. 8 ans et M. 5 ans).

\section{Transmettre des connaissances}

10 Visiter un musée de beaux-arts en famille, c'est aussi et surtout interagir face à des œuvres d'art, avec la plupart du temps le désir chez l'adulte de transmettre à son enfant des connaissances que lui même ne possède ou ne maîtrise pas, ou peu. En effet, rappelons qu'aucun des parents rencontrés ne considère détenir de bonnes connaissances en histoire de l'art. Face à ces lacunes que tous évoquent, on observe plusieurs attitudes. 
La visite en famille permet de transmettre des connaissances à son enfant.

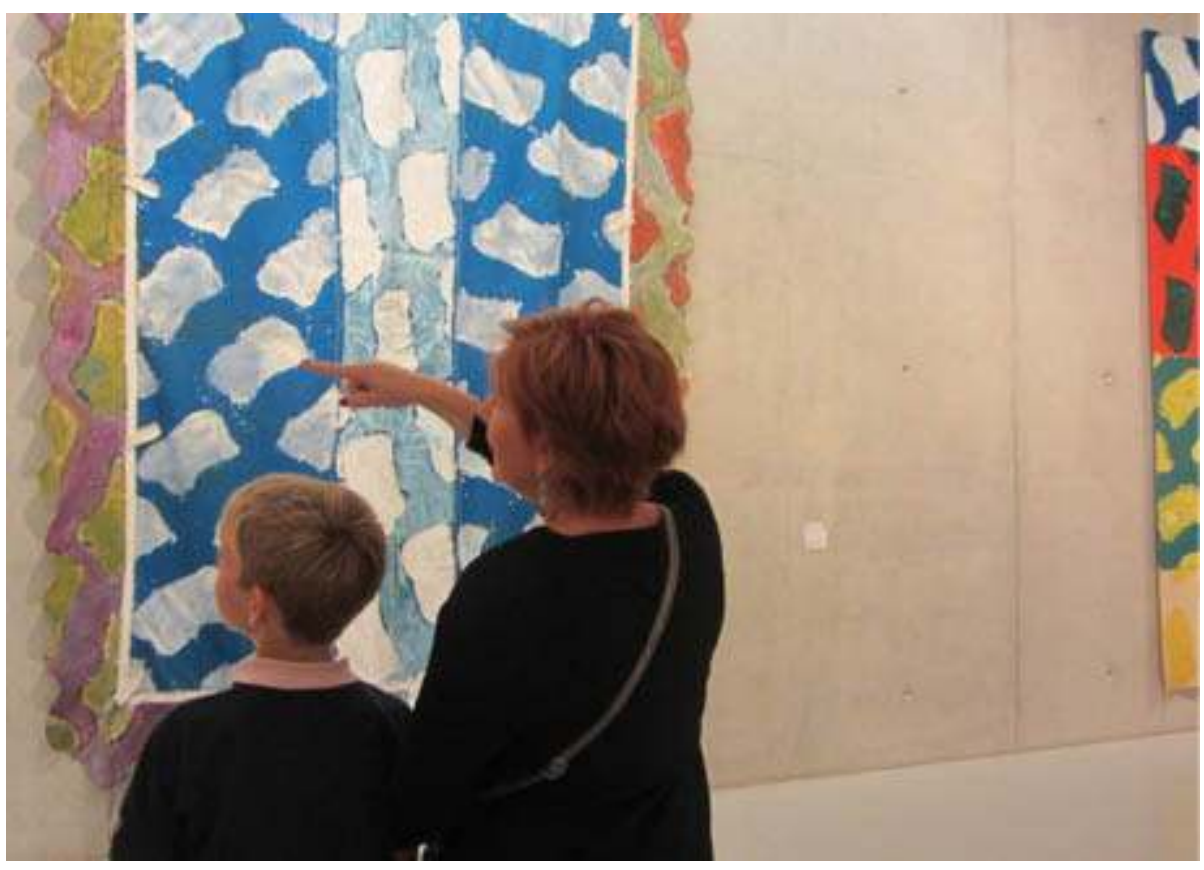

(C) Aude Joly

\section{La crainte de ne pas "être à la hauteur"}

11 Lorsque l'objectif de visite est avant tout éducatif, le parent se sent rapidement démuni voire angoissé face à son incapacité à répondre aux questions de son enfant: "c'est vrai qu'ils posent des questions et qu'il faut savoir répondre. J'essaye d'expliquer, des fois ils me posent des colles, je ne sais pas répondre" (mère de R. 7 ans). Face à cette crainte de ne "pas être à la hauteur" les parents développent diverses stratégies: stratégie d'évitement pour ces parents qui préfèrent ne pas fréquenter les musées avec leurs enfants "ce qui nous manque aussi, c'est la connaissance, [...] c'est difficile d'amener les enfants quand on ne sait pas quoi leur dire" (mère de M. 8 ans) ; stratégies d'évitement du statut de parent médiateur "je suis très mauvaise pédagogue, du coup pour lui expliquer des trucs, je préfêre même me taire, ne rien lui dire, que de rentrer dans des explications" (mère de L. 10 ans) ; stratégies de préparation. Il peut s'agir pour le parent de s'informer "il faut se préparer, connaître les thèmes, parce que tout est expliqué sur le site du musée : les thèmes, les salles" (mère de R. 7 ans), ou de préparer l'enfant "à la médiathèque, j'ai pris un livre sur le musée Fabre, et un livre sur Soulages. Pour la préparer à voir Soulages, des monochromes" (mère de M. 7 ans). Seules deux mères expliquent avoir recours à des médiations spécifiques (visites guidées ou livrets jeux) et une regrette ne jamais avoir pris le temps de participer aux visites en famille proposées très régulièrement par le service des publics du musée Fabre. Ces stratégies, qu'elles soient de préparation, d'évitement ou de délégation (en faisant appel à des médiations proposées par les musées), illustrent les travaux de Lakota dont les résultats ont montré que les adultes accompagnés d'enfants ont tendance à choisir des thématiques de visite qui leur sont familières et dont ils maîtrisent le contenu ${ }^{4}$. Le cas des salles Soulages est une bonne illustration de ce type de comportement. Rappelons que dans le cadre de cette enquête, les familles sont libres d'adopter le rythme qu'elles souhaitent, mais que les salles visitées leur sont imposées. Le travail du peintre aveyronnais est largement considéré par les 
parents comme un art difficilement accessible pour des enfants. L'attitude de ce père est une des plus marquées mais rejoint l'opinion d'une majorité de parents :

"P : Ça n'est pas une expo où je l'aurais amenée. Naturellement, j'aurais même boycotté [...] pour éviter que ce soit compliqué, c'est quand même spécial.

I : Vous n'auriez pas su comment faire?

P : Ah non pas du tout. Je pense que je me serais dit qu'elle s'ennuierait beaucoup."

(père de A. 10 ans)

Il est intéressant de constater que cette volonté pour le parent de donner à la visite en famille un objectif éducatif qu'il se sent le devoir d'assumer n'est pas présente à la fois chez les familles dont la pratique de visite est régulière et correspond à une pratique socialement inscrite dans l'histoire familiale, et chez les familles pour qui cette expérience de visite était la première. Il semblerait que pour un parent, découvrir pour la première fois, au même titre que son enfant, un lieu et une pratique inconnu le "libère" de la pression éducative perçue par d'autres.

\section{Le manque d'informations}

Visiteurs en famille suivant une visite par audioguide, musée Fabre, Montpellier Agglomération.

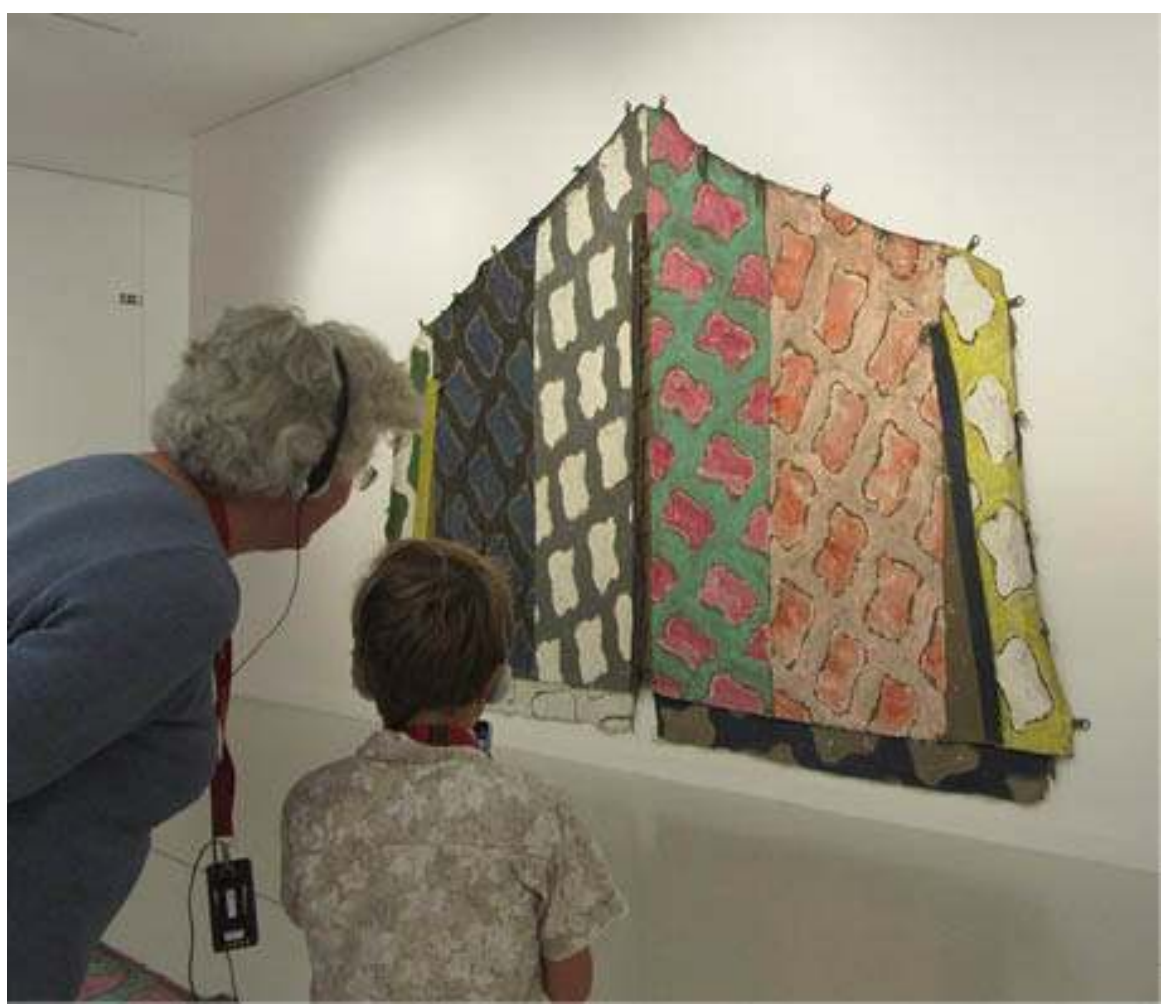

(c) Musée Fabre, Montpellier Agglomération

En plus de dire ce sentiment de ne pas "être à la hauteur" pour commenter les œuvres, certains adultes regrettent de ne pas trouver assez d'informations adaptées à leur condition de parents dans les salles du musée et expriment le sentiment d'être "livrés à eux-mêmes" dans les salles d'exposition. Face à leur désir de transmettre des connaissances à leurs enfants, certains parents se sont tournés vers les fiches de salles (rappelons que le musée Fabre propose aux visiteurs en familles divers livrets de visite, mais que pour les besoins de l'étude, aucune de ces médiations spécifiques n'a été remise 
aux familles participantes). Les parents ayant eu recours aux fiches de salles considèrent cet outil comme peu voire pas adapté à leurs besoins, soulignant le fait qu'elle "sont faites pour des adultes" (mère de L. 9 ans), et regrettent le fait que le musée ne propose pas l'équivalent pour un public familial: "il faudrait des fiches vraiment plus simples, et moins longues, pour les parents. Parce que quelqu'un qui ne sait pas trop, c'est compliqué quand même, pour en plus expliquer à son enfant" (mère de T. 7 ans). Une autre mère exprime de manière encore plus précise ce qu'elle attendrait d'une médiation écrite adapté aux familles : "on pourrait imaginer des notices explicatives supplémentaires pour les enfants, avec une question à leur poser par tableaux. [...] Là c'est fait pour les adultes, on le voit" (mère de E. 8 ans). Si cette demande est récurrente chez les familles rencontrées, on peut s'interroger sur l'utilité que ce type de médiation aurait pour les familles si elles étaient mises en place par les musées. En effet, l'étude menée au musée Dauphinois sur l'utilisation des textes enfants montre que les adultes n'utilisent que "très peu, voire pas du tout les dispositifs de médiation de l'exposition" (Duclos, Philippeaux et Poli, 2010).

\section{Transmettre le goût d'une pratique}

\section{La crainte de susciter un rejet}

14 Au-delà de la transmission de notions d'histoire de l'art, la visite en famille aurait pour objectif de transmettre un goût pour la pratique muséale et pour la contemplation d'œuvres d'art. Il semblerait que plus la pratique muséale est ancrée dans la vie de la famille, moins ces visites prennent l'aspect d'une pratique éducative. Pour une petite minorité de parents, la visite d'un musée a en effet davantage l'objectif de transmettre un habitus, d'échanger et de partager des émotions et des opinions. Dans ce cas là, le parent ne ressent pas ou moins l'obligation de commenter les œuvres à son enfant. Pour cette mère familière des visites en famille, il n'est pas nécessaire, voire pas recommandé d'apporter des notions d'histoire de l'art à sa fille "c'est le meilleur moyen d'ennuyer les enfants en leur disant : le peintre a fait ça, dans tel contexte historique. Si le musée devient un lieu de leçon, un lieu d'apprentissage, on n'y retourne pas de gaîté de coeur. [...] Donc je n'explique pas, et puis moi je ne suis pas spécialisée en peinture, je ne suis pas capable de faire ça". Elle développe par contre lors de ces visites des activités pour impliquer sa fille, éduquer son regard: "on faisait des jeux, [...] celui qui avait gagné, c'est celui qui avait vu le plus de chiens. C'est différent parce qu'il y a un côté ludique. [...] Forcément, vous regardez le tableau, et vous voyez forcément la différence entre le chien qui apparaît dans un tableau du XIXe, et celui qui apparait dans un tableau de la Renaissance, sans qu'il y ait besoin de formulation d'adulte, on n'a pas besoin de vous expliquer que le chien n'est pas peint de la même manière" (mère de J. 10 ans).

On retrouve ce type de jeux que l'on pourrait qualifier de "stratégies de médiation parentale", dans les récits d'autres parents, que ce soit sous la forme de questions/ réponses ou de dessins proposés devant les œuvres. Ces stratégies pensées à l'avance ou improvisées permettent non seulement d'allier le plaisir de l'adulte et celui de l'enfant pour une activité qu'il n'a souvent pas choisie, et placent clairement les adultes dans une attitude active, voire participative, de visite : ils mènent la visite et se positionnent en médiateur du musée auprès de leurs enfants. Ces stratégies nous semblent révélatrices du statut de "guide" ou de "médiateur" que le parent adopterait de manière consciente ou non. Même si elle ne demande pas de connaissances particulières, cette position est, chez certains parents source d'appréhension. La peur que l'enfant ne s'ennuie, qu'il n'ait rien 
d'autre à faire que de "regarder", que la visite soit trop longue, amène chez certains parents la crainte de lui transmettre, plutôt qu'un goût, une aversion pour le musée : " imaginez, nous on vient en famille, on ne connaît rien sur les peintres, il n'y a pas de thématique précise, on n'organise rien de spécial. Je me dis qu'au lieu de les sensibiliser au musée, je vais les en dégoûter. Quand on n'est pas des spécialistes c'est très difficile" (mère de A. 7 ans).

Un atelier en famille organisé au musée Fabre.

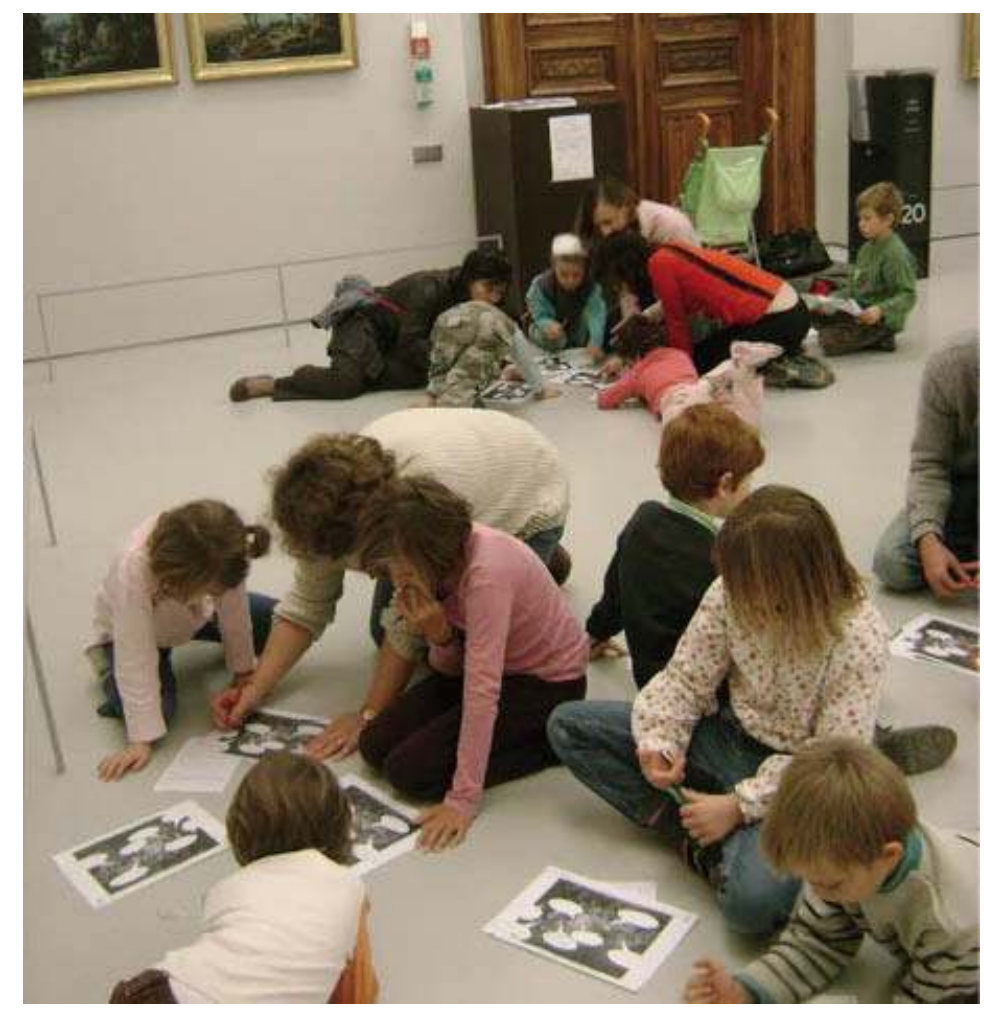

(c) Aude Joly

\section{Le manque d'approche sensible}

Les parents sont parfois donc démunis et demandeurs de conseils de la part du musée pour transmettre un goût pour une pratique, quelles sont les œuvres à privilégier? Que faire lorsque l'attention de l'enfant décroît? Comment trouver d'autres approches que la simple parole ? Là encore, les familles regrettent le manque d'outils dans le musée pour les aider à partager une expérience esthétique et émotionnelle avec leurs enfants : " il pourrait y avoir un petit coin pour les enfants, ça serait sympa [...] d'avoir un petit contrepoint, où à son tour on peut manipuler, créer..." (mère de L. 9 ans et T, 7 ans). Un lieu qui permettrait d'aborder les œuvres autrement que par le regard ou la simple parole est parfois souhaité : "je pense qu'un côté tactile serait intéressant notamment sur des Soulages. Elle avait envie, elle a quasiment mis le doigt dessus à un moment! Les adultes aussi, on a envie de toucher, donc un lieu, pour les plus petits où on puisse toucher" (mère de A. 9 ans). 


\section{Être confrontés à des œuvres "inadaptées"}

17 Au delà de la transmission d'un savoir, certaines familles ont ressenti lors de cette visite ou de visites passées des difficultés d'un autre ordre face à certaines œuvres. Visiter un musée de beaux-arts en famille c'est en effet déambuler dans des espaces inconnus, que l'on découvre au détour d'une salle, d'un couloir. Cette découverte non préparée des expositions peut amener l'adulte à aborder des questions beaucoup plus larges que celles relatives à l'histoire de l'art ou à la démarche d'un artiste. Une des familles rencontrées exprime bien la difficulté que père et mère ont éprouvée face à certains tableaux dont l'iconographie renvoie à des sujets qu'ils estiment délicats à aborder avec leur fille de 7 ans. Par pudeur tout d'abord, le père a préféré poursuivre la visite de son côté dans certaines salles, laissant sa femme et sa fille observer seules les œuvres représentant des nus, ce qui n'a pas empêché la famille de dialoguer et d'échanger lorsqu'ils se croisaient dans les salles :

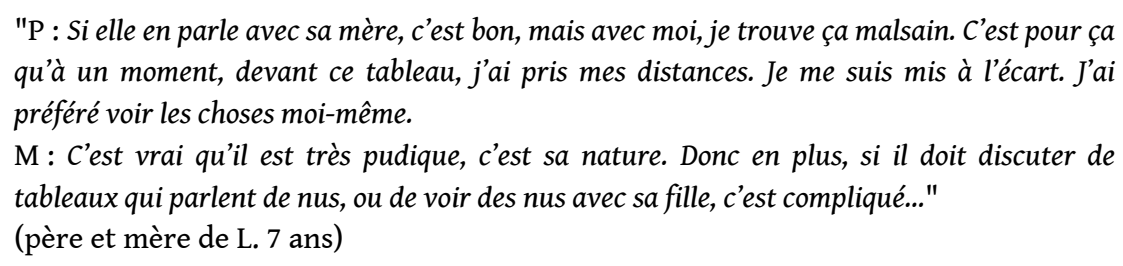

D'autres œuvres ont éveillé chez sa mère la crainte que les sujets rencontrés ne l'effraient, soulevant des questionnements qu'elle n'a pas l'habitude d'aborder avec sa fille (mort, religion). Soulignant l'intérêt d'une visite de musée pour avoir l'occasion d'aborder ces sujets, cette mère ne minimise pas l'impact que cela pourrait avoir sur sa fille, tandis que son père explique qu'il se sentirait plus à l'aise dans un musée où les choix d'accrochage de certaines salles seraient en quelque sorte "adaptés" à la visite familiale, avec des sujets enfantins, moins graves.

\footnotetext{
"M : Il y a un tableau, je ne sais pas si ça lui a fait peur, mais elle s'est demandé si ça existait vraiment l'Enfer et le Paradis. J'ai vu un changement radical quand elle l'a regardé. C'était un mélange de peur et de questionnement. Ça oblige aussi à se poser certaines questions, il y a des thèmes qu'on n'aborde pas avec les enfants, et avec les tableaux, on peut les aborder. Je sais qu'elle va se poser des questions pendant très longtemps pour savoir si ça existe vraiment..."

(mère de L. 7 ans)
} 
Livret jeu pour les familles au musée Fabre, Montpellier Agglomération.

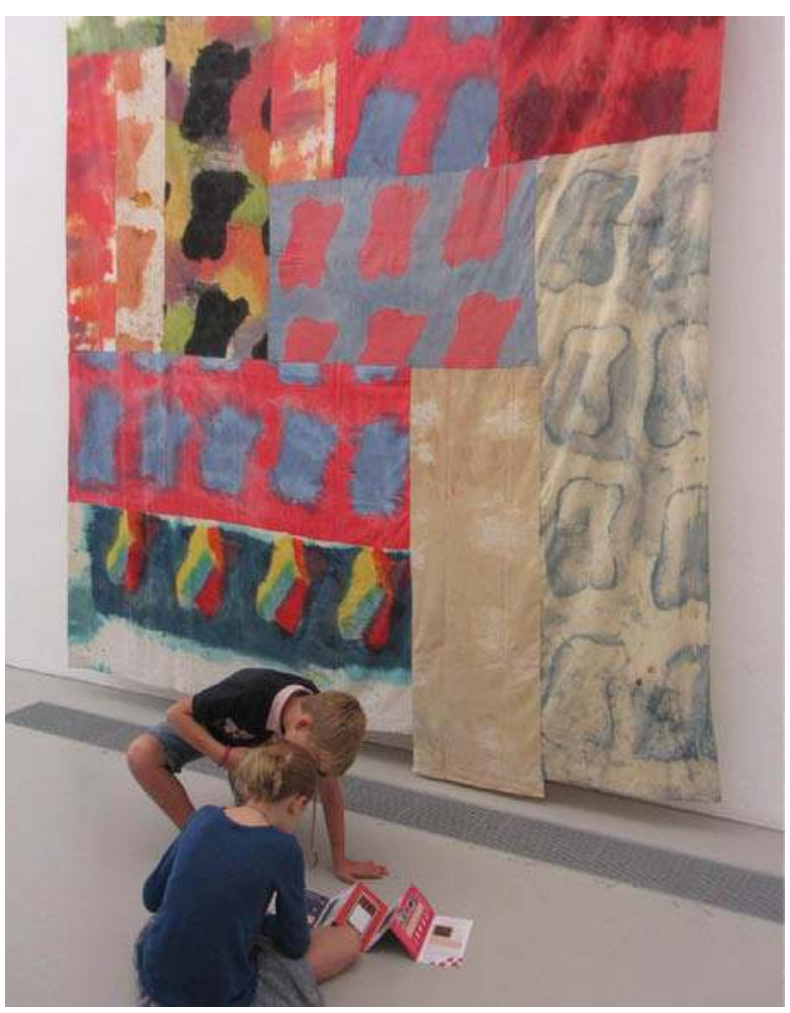

(c) Aude Joly

Une autre mère, qui visite très régulièrement les musées et lieux d'exposition d'art avec sa fille, explique aussi avoir été confrontée à ce type de difficultés lors d'une visite antérieure. Le caractère exceptionnel d'une exposition temporaire présentant au musée Fabre des œuvres de Caravage l'a poussée à montrer à sa fille des tableaux qu'elle estime après coup peut-être trop durs pour son âge.

M : Caravage, c'était glauque, alors je l'avais prévenue [...] mais les têtes coupées, c'était difficile. [...] J'avoue que si ça avait été des tableaux qui soient tout le temps à Montpellier, j'aurais peut-être attendu un peu. Une fois sur place, j'ai vu sa réaction, tout de suite elle s'est cachée derrière ma main"

(mère de M. 7 ans).

\section{Conclusion et perspectives : la pratique scolaire des enfants pour une inversion des rôles?}

Les résultats de cette étude visant à connaître les difficultés auxquelles sont confrontés les parents qui visitent un musée de beaux-arts avec leur enfant révèlent que le statut d'accompagnateur du parent le positionne dans un rôle de médiateur parfois difficile à assumer. Soucieux de transmettre un goût pour une pratique culturelle aujourd'hui très valorisée, les parents éprouvent des difficultés concernant les codes de comportement à faire respecter, mais aussi expriment les blocages qu'ils ressentent face à leur manque de connaissances en histoire de l'art. Le manque d'informations, notamment de textes adaptés à leur situation de transmetteur est souvent regretté, et les médiations spécifiquement conçues par les musées pour le public familial restent méconnues. 
Il est intéressant toutefois de noter que ce rôle de parent-médiateur, souvent difficile à assurer pour les adultes, peut être repensé au regard de la pratique scolaire des enfants. En effet, cet article s'inscrit dans une recherche plus large, visant à analyser les changements de comportement au sein de la visite en famille lorsque cette dernière se passe après que l'enfant soit venu au préalable avec sa classe. Ainsi, sur les 26 familles participantes, 9 ont visité le musée après que leur enfant a suivi une visite scolaire intitulée "Le noir-lumière de Pierre Soulages". Pour cet échantillon restreint, l'entretien mené au terme de la visite a porté sur les différences et les éventuelles surprises que les parents ont pu ressentir, en comparaison avec leurs expériences de visite passées. Le fait de visiter avec son enfant un musée de beaux-arts après que celui-ci ait suivi une visite scolaire peut-il entraîner une situation d'inversion des rôles et réduire les appréhensions du parent en visite en famille?

Pour les 9 familles venues visiter le musée dans ce cadre, il est intéressant de relever les changements d'attitudes et de comportements que ces adultes ont développés au cours de cette visite familiale. Il apparait que la visite scolaire a un réel impact sur les modes de communication et le statut que parents et enfants adoptent lors d'une visite en famille. L'expression "inversion des rôles" est employée à plusieurs reprises par les familles lorsqu'elles relatent leur expérience de visite dans les salles Pierre Soulages, connues de leurs enfants. La situation de transmission exceptionnelle est évoquée de manière très positive par les familles : "d'habitude c'est toujours les grands qui apprennent aux petits. Et sur des choses, finalement, peut-être subjectives, sur des sentiments, sur des impressions, c'est l'enfant qui va transmettre à l'adulte, c'est important pour un enfant" (mère de J. 10 ans). "Des fois c'est pas que le parent qui apprend des choses aux enfants, des fois c'est les enfants qui apprennent aux parents. [...] ça m'a fait plaisir, pour une fois, les rôles se sont inversés et c'était sympa" (mère de R. 7 ans). Ils estiment non-seulement avoir appris de leur enfant, mais lui reconnaissent volontiers un statut qui pourrait s'apparenter à celui de connaisseur, de "guide", ou de "déjà initié". Certains d'entre eux apprécient le fait d'être en quelque sorte "déchargés" de leur statut habituel de parent-médiateur : "là c'était elle qui était guide. Ce n'est pas à moi de présenter un tableau. Non, non, là c'est [elle] qui me, comment dire, qui m'apprend. C'est bien" (mère de J. 10 ans). Cette situation d'apprentissage inversé génère en outre chez les parents un sentiment d'étonnement mêlé de fierté comme par exemple cette mère, surprise que sa fille commente autant les œuvres que leur accrochage et la scénographie : "j'étais surprise de voir tout ce qu'elle avait retenu, ou savais, je sais pas après d'où vient ce qu'elle me disait. Parce que, je trouvais qu'elle avait vraiment une bonne connaissance et elle m'a surprise parce qu'elle ne regardait pas que l'œuvre, mais l'œuvre dans le musée. L'éclairage... quand même, c'est pas mal!" (mère de L. 7 ans). 
Dessin dans les salles Soulages au musée Fabre, Montpellier Agglomération.

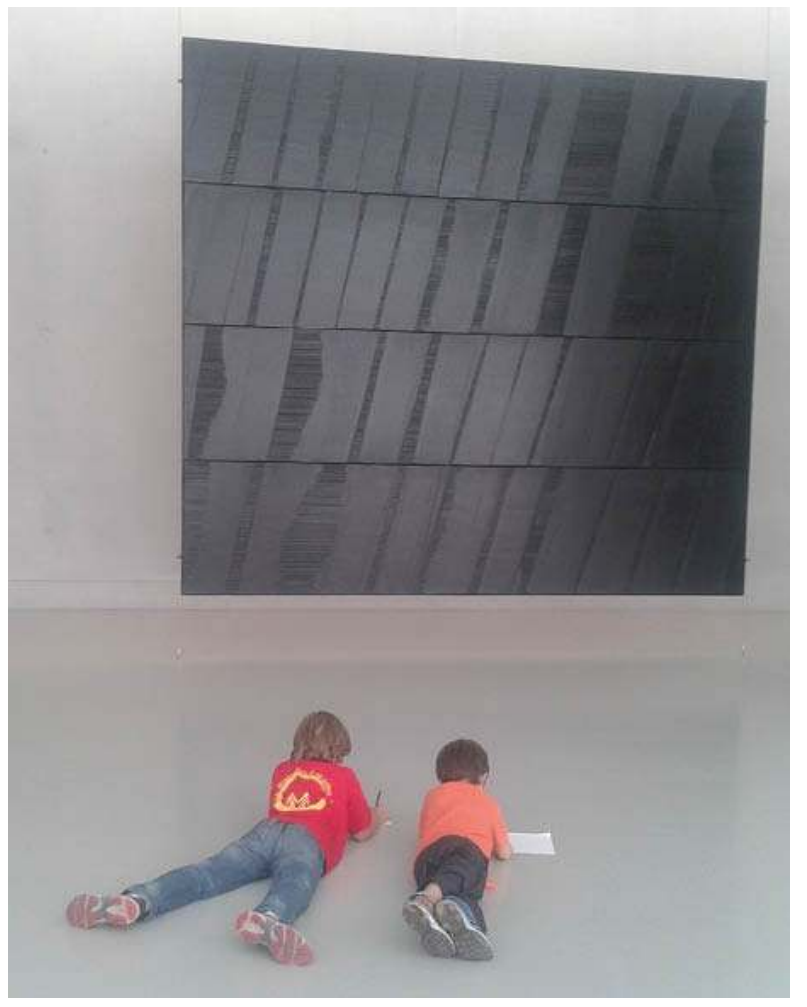

(c) Aude Joly

Au regard des difficultés relevées plus haut, il est intéressant de noter que pour cette visite, cette situation de transmission inversée annule les appréhensions des parents. Guidés par leur enfant dans un lieu et des salles qu'il ou elle connaît, les parents n'évoquent pas les difficultés liés aux comportements de visite. Pour une famille dont les parents redoutent particulièrement l'attitude de leur enfant lors de visites de musées, c'est pour eux la "mission" de guide endossée par leur fils, le fait qu'il soit intéressé et actif, qui l'a conduit à se comporter de manière adaptée. En outre, une visite en famille se déroulant après une visite scolaire permet aux parents de découvrir avec leur enfant des œuvres qui considéraient de prime abord difficiles d'accès. Le cas des salles Soulages est particulièrement révélateur. Les parents, qu'ils connaissent ou non son œuvre et qu'ils y soient ou non sensibles ont tous été surpris de l'intérêt que leur enfant y portait. Le fait que l'enfant les connaisse, les commente, raconte sa visite, lève pour le parent la difficulté liée à trouver la manière de l'intéresser à des œuvres abstraites (Joly, à paraître).

24 Ces éléments viennent nourrir l'hypothèse supposant que l'enfant, en passant du statut d'élève-visiteur à celui de fils/fille, endosserait auprès de ses parents le rôle de guide du musée et des œuvres. L'expérience de visite dans le cadre scolaire de l'enfant aurait donc une réelle influence sur le rôle et le statut du parent et de l'enfant lors de la visite en famille et semble considérablement limiter les appréhensions et les difficultés rencontrés par les parents lors de visites de musées en famille. En prenant parfois en compte le désir de l'enfant et en lui conférant un rôle moteur dans l'acte de visite, l'adulte et l'enfant se retrouveraient dans des rôles de transmission quasi-inversés qui suggère une rupture avec la logique traditionnelle de la transmission. "Les parents, n'étant pas toujours des guides, 
doivent rendre visible cette rupture avec la définition du parent pédagogue, du parent professeur, du parent transmetteur" (De Singly, 2003).

Les liens entre visite scolaire et visite en famille restent donc particulièrement intéressant à creuser pour les musées de beaux-arts, et les dispositifs parfois qualifiés de "passerelles" consistant à inviter les familles des élèves visiteurs au musée pour une visite préparée et menée par eux méritent en ce sens d'être l'objet de futures études.

\section{BIBLIOGRAPHIE}

Benneteu, B., Albertin, A.-M., Hue, M., Pelletier, A. et Rooryck, I. Adams, M. et Moussouri, T. The interactive experience: linking research and practice. Actes du colloque Interactive Learning in Museums of Art and Design, Victoria \& Albert museum, 2002.

Cordier, J.-P. La transmission familiale au musée, in Allard, M. et Lefebvre, B. (dir.) Le musée, un lieu éducatif. Montréal : Musée d'art contemporain, 1997, pp. 188-199.

Debenedetti, S. Visites occasionnelles du musée d'art et confort de visite : le rôle des compagnons, in Donnat, O. et Tolila, P. (dir.) Le(s) public(s) de la culture. Paris : Presses de Science Po, 2003, pp. 273-278.

De Singly, F. La famille individualiste face aux pratiques culturelles, in Donnat, O. et Tolila, P. (dir.) Le(s) public(s) de la culture. Paris : Presses de Science Po, 2003, pp. 43-59.

Duclos, A., Philippeaux, F. et Poli, M.-S. De l'utilité du "texte enfant" au musée, La Lettre de l'OCIM, $\mathrm{n}^{\circ} 132,2010$, pp. 28-33.

Joly, A. Découvrir en famille les œuvres de Pierre Soulages après une visite scolaire, in Muséologies, cahier d'études supérieures, Montréal, à paraître.

Jonchery, A. Quand la famille vient au musée : des pratiques de visites aux logiques culturelles. Thèse de doctorat, Muséum national d'Histoire naturelle, 2005.

Jonchery, A. Se rendre au musée en famille, La Lettre de l'OCIM, $\mathrm{n}^{\circ} 115,2008$, mis en ligne le 10 novembre 2010. URL : http://ocim.revues.org/264

Octobre, S. Connaître les populations et les publics, in Eideilman, J., Roustan, M. et Goldstein, B. (dir.) La place des publics. De l'usage des études et recherches par les musées. Paris : La Documentation française, coll. "Musées-mondes", 2008, pp. 91-105.

Octobre, S. et Jauneau, Y. La transmission culturelle : tels parents, tels enfants ? La revue française de sociologie, $\mathrm{n}^{\circ}$ 49-4, 2008, pp. 695-722.

Pasquier , D. La culture comme activité sociale, in Maigret, E. et Macé, E. (dir.) Penser les médiacultures. Paris : Armand Colin, 2005. 


\section{NOTES}

1. Afin d'alléger le texte, les mots «"parent" et "enfant" sont utilisés au singulier de manière générique.

2. Nous souhaitons vivement remercier la direction du musée Fabre qui, en accordant la gratuité aux familles participantes, a largement contribué à faciliter la réalisation de cette étude, ainsi que l'équipe de médiation et de surveillance sans qui l'enquête de terrain n'aurait pas pu avoir lieu.

3. $\mathrm{M}$ : mère ; $\mathrm{P}$ : père, $\mathrm{I}$ : interviewer

4. Voir à ce propos Schiele, B. et Koster, E. (dir.) La révolution de la muséologie des sciences : vers les musées du XXIe siècle. Lyon : Presses universitaires de Lyon, Québec : Éditions Multimondes, collection Muséologies, 1998, pp. 258-259.

\section{RÉSUMÉS}

Cette étude du comportement des parents et des enfants lors d'une visite du musée en famille dans laquelle prédomine une logique de transmission éducative - rend compte des difficultés auxquelles sont confrontés les parents dans leur rôle de médiateur et fait le constat que lorsque la visite scolaire précède la visite en famille, la situation de transmission est inversée avec un passage du parent-médiateur à l'enfant-guide.

\section{INDEX}

Mots-clés : musée, visite, famille

\section{AUTEUR}

\section{AUDE JOLY}

Professionnelle de la médiation muséale, doctorante en muséologie, laboratoire Culture et Communication, université d'Avignon et des Pays de Vaucluse aude.joly84@gmail.com 